\title{
Claves de Compround para la redefinición del modelo de evaluación de la calidad docente en la Universidad de Concepción
}

\author{
Keys of Compround to redefine the model of evaluation of the quality \\ of teaching at University of Concepción \\ Ignacio González Lópeza, Ana López Cámara ${ }^{b}$, Ignacio Nail Kroyer \\ a Universidad de Córdova, España. Correo electrónico: ignacio.gonzalez@uco.es \\ b Universidad de Córdova, España. Correo electrónico: anabelen@uco.es \\ c Universidad de Concepción, Chile. Correo electrónico: onail@udec.cl
}

\begin{abstract}
RESUMEN
Este artículo muestra las aportaciones del modelo competencial Compround, que recoge los resultados de una investigación realizada en España cuyo propósito fue generar la figura del buen docente universitario, y su conexión con el sistema de evaluación del profesorado y posterior definición del perfil docente de la Universidad de Concepción. Partiendo del análisis de la información recabada a través de esta institución chilena acerca del proceso de evaluación docente y sobre las bases teóricas y prácticas que presenta el modelo Compround, se presentan una serie de propuestas que reviertan en el logro de mejores situaciones de aula que propicien el éxito académico y profesional del alumnado.
\end{abstract}

Palabras clave: competencias, perfil docente, evaluación de la docencia, grupo de discusión.

\begin{abstract}
This article shows the contributions of the Compround model, which collects the results of an investigation conducted in Spain, whose purpose was to generate the figure of the good university teaching and, its connection with the system of evaluation of teaching staff and the subsequent definition of the teaching profile of University of Concepcion. Based on an analysis of the information of the evaluation process, collected by this Chilean institution, and on the theoretical and practical bases that the Compround model involves, a number of proposals, aiming for better situations in the classroom and facilitating academic success and professional success in students, are presented.
\end{abstract}

Key words: skills, teaching profile, evaluation of teaching, focus group 


\section{INTRODUCCIÓN}

La evaluación de la actividad docente del profesorado universitario es uno de los pilares esenciales en los que las instituciones de educación superior inciden actualmente con el fin de obtener resultados exitosos en un proceso de enseñanza y aprendizaje adaptado a modelos educativos basados en competencias, cuya finalidad es la incorporación de sus estudiantes a la vida social, económica y cultural con altos niveles de eficiencia. Se destacan como elementos de valoración, entre otros, el alumnado, autogestión del aprendizaje, trabajo colaborativo, estrategias de enseñanza y tareas de aprendizaje, evaluación acorde con la consecución de unas competencias generales y específicas, etc. (González y López, 2010: 404).

Sin embargo, a pesar de su relevancia manifiesta, expuesta en múltiples foros, no existe aún un consenso de lo que se puede denominar la figura del "buen profesor" o "buena profesora" universitaria en referencia al establecimiento de un modelo de docente deseado. Como ya apuntaron Tejedor y García Valcárcel (1996), la evaluación de la docencia y del subsistema profesorado es aún hoy en día una herramienta con importantes limitaciones, no solo de carácter teórico (diversidad de finalidades y carencia de modelos de profesor ideal), sino también de carácter práctico, ya que es difícil elegir la estrategia evaluativa adecuada, puesto que su validez ha de establecerse indirectamente ante la carencia mencionada de un modelo epistemológico que delimite con claridad los parámetros que definen el constructo "calidad docente" y, además, se adapte a la idiosincrasia de cada institución universitaria.

Lo que sí tenemos claro respecto a la evaluación del profesorado es que se trata, como bien señaló Tejedor, “de un proceso que debe orientarse fundamentalmente a la estimación del nivel de calidad de la enseñanza a fin de contribuir progresivamente a su mejora. El criterio básico será conseguir una utilidad efectiva del conjunto del proceso como recurso de perfeccionamiento docente haciendo buenos los propósitos de la evaluación formativa" (2012: 19).

\subsection{LA EVALUACIÓN DE LA ACTIVIDAD DOCENTE DEL PROFESORADO}

Evaluar la labor docente del profesorado universitario es un proceso que busca obtener evidencias para poder emitir juicios de valor sobre el cumplimiento de las responsabilidades del docente en el proceso de enseñanza-aprendizaje y en el desarrollo de las competencias de sus estudiantes, todo ello a partir de información válida, objetiva y fiable, con el último propósito de facilitar la toma de decisiones en relación a los diferentes elementos que definen al propio profesional y a su trabajo con el alumnado (Tejedor y Jornet, 2008).

Esta evaluación debe tener en cuenta las funciones específicas de la labor docente, que Tejedor (2012) expresa en dos categorías que recogen los saberes, habilidades, actitudes y valores inherentes a esta función profesional:

- $\quad$ El saber: ¿Sabe lo que enseña? ¿Sabe cómo enseñarlo? ¿Conoce los procesos de desarrollo y aprendizaje de sus estudiantes?

- El hacer: ¿Hace lo que se ha comprometido? ¿Lo hace bien? ¿Es respetuoso con sus estudiantes?

La primera categoría hace referencia al dominio de los saberes (dimensión competencial de carácter epistemológico y cognitivo) y la segunda al dominio de las conductas (dimensión competencial de carácter procedimental y actitudinal). 
Más allá de esta doble dimensión competencial docente del profesorado universitario desde una perspectiva teórica, Zabalza (2007: 106) relata desde la práctica las tres dimensiones en las que se configura este rol profesional y que, por tanto, inciden de un modo más sistemático en el compendio de competencias que constituyen la figura del profesor o la profesora y que deben ser, por tanto, objeto de los procesos de evaluación que aquí se tratan de avanzar:

1. Dimensión profesional, que permite acceder a los componentes clave que definen ese trabajo o profesión: cuáles son sus exigencias, cómo y en torno a qué parámetros constituye su identidad profesional, cuáles son los principales dilemas que caracterizan el ejercicio profesional en ese ámbito, cuáles son las necesidades de formación inicial y permanente, etc.

2. Dimensión personal, que permite entrar a considerar aspectos de gran importancia en el mundo de la docencia: tipo de implicación y compromiso personal propio de la profesión docente, ciclos de vida de los y las docentes y condicionantes de tipo personal que les afectan, problemas de tipo personal asociados al ejercicio docente, fuentes de satisfacción e insatisfacción en el trabajo y la carrera profesional.

3. Dimensión laboral, que nos sitúa ante los aspectos más claramente relacionados con las condiciones contractuales, los sistemas de selección y promoción, los incentivos y las condiciones laborales de la profesión docente.

Más allá de estas aportaciones, los trabajos realizados por Saravia (2011) aportan un modelo estructural de competencias del profesorado universitario que se configura a partir de las cualidades que ha de poseer un profesional para lograr la excelencia en la docencia. Estas son: la competencia científica (demostración de los conocimientos propios de la formación disciplinar), la competencia práctica (conocimientos y criterios procedimentales e instrumentales que permiten el desarrollo correcto de las actividades), la competencia personal (posición subjetiva del profesorado respecto al saber) y la competencia social (cualidades que permiten establecer relacionales interpersonales socialmente aceptadas).

Se puede comprobar, de este modo, que un sistema de evaluación de la docencia debe abarcar un conjunto de mecanismos que permita definir el grado en que las personas contribuyen al logro de los estándares requeridos para el cargo o puesto que ocupan en la organización, así como para los objetivos de la institución, como expresó en su momento Fuchs (1997). Ello podrá facilitar el diseño de las medidas necesarias para que el profesorado tenga oportunidades de desarrollo profesional y personal dentro de la universidad en la que ejerce su trabajo.

La mayoría de las universidades incorporan sistemas de evaluación de la excelencia docente en base a la opinión que manifiesta el alumnado de la labor ejercida por el profesorado en el desarrollo y nivel de adquisición de las competencias a emplear en el entorno profesional y en los ámbitos social y personal. No pocos expertos opinan que este es el mejor procedimiento para llevar a cabo esta evaluación, pues el alumnado es el permanente observador de la actuación de su profesorado (Tejedor, 2003). Su práctica se basa, principalmente, en la aplicación de un cuestionario que, como veremos en el caso de la Universidad de Concepción (UdeC), está siendo objeto de análisis y adaptación a los cambios sugeridos por el nuevo modelo docente definido en la institución (Universidad de Concepción, 2015). 
En definitiva, la implementación de estas propuestas no debe suponer la aplicación de una serie de pruebas llevadas a cabo puntualmente, sino un proceso continuo y sistemático encaminado, desde el punto de vista de Jornet, Villanueva, Suárez y Alfaro (1988), a ayudar a cada uno de los y las docentes en su desarrollo profesional y en la planificación de su carrera, así como contribuir a que la formación del alumnado se ajuste a las necesidades reales del entorno profesional y social en el que se inserta. Como valor añadido, incorporamos la excelencia que la universidad entiende que supone disponer de un profesorado cualificado y un alumnado satisfecho con la labor ejercida en sus aulas.

\subsection{EL MODELO COMPROUND PARA LA EVALUACIÓN DE LAS COMPETENCIAS DEL PROFESORADO UNIVERSITARIO}

Compround (Competencias del Profesorado Universitario) se concibe como un modelo de evaluación financiado bajo el auspicio de la Dirección General de Universidades del Ministerio de Educación español (EA2009-0005) con el propósito de definir las competencias docentes que las universidades consideran esenciales para definir el rol del profesorado universitario y, a partir de su análisis, establecer un sistema de indicadores de evaluación de la calidad docente, en el sentido en que son aceptados por su carácter sintético y su capacidad para orientar la toma de decisiones.

Las diferentes unidades técnicas de calidad de las instituciones universitarias españolas de carácter público aportaron los instrumentos en uso para llevar a cabo la evaluación docente de su profesorado, siempre desde la percepción manifestada por el alumnado. Era de suponer que dichos sistemas contaban con las garantías científicas pertinentes de fiabilidad y validez, así como de adaptación a las características contextuales, sociales y educativas de las diferentes universidades.

A partir de las dimensiones analizadas, se identificaron las competencias a valorar y se determinaron los indicadores pertinentes para cada una de ellas, en función de los cuales procedimos a la construcción de un protocolo de evaluación de la labor docente del profesorado universitario, presente en González y López (2010).

Fueron dos los momentos de relevancia que dieron como resultado la elaboración del protocolo de evaluación citado. El primero de ellos consistió en el análisis exhaustivo de la información recogida de los instrumentos que nos aportaron las diferentes universidades, comenzando por su denominación, así como por un estudio de los sistemas de medición empleados, las dimensiones consideradas y los indicadores desarrollados. En un segundo momento, y tras la construcción de un borrador, este fue sometido a valoración por parte de una serie de expertos y expertas de cinco universidades que procedieron a estimar la pertinencia y claridad de los elementos propuestos, así como la definición e idoneidad de las dimensiones en los que estos fueron agrupados.

Los indicadores establecidos por parte del grupo de expertos y expertas fueron convertidos en ítems de valoración escalar, dando lugar a una encuesta que se implementó a un total de 1316 estudiantes a quienes se solicitó su valoración, lo que permitió construir un modelo de evaluación de las competencias docentes del profesorado universitario.

Los diferentes análisis exploratorios realizados sobre la información obtenida (descriptivos, inferenciales y multivariados) dieron como resultado un protocolo de 41 indicadores de evaluación competencial docente agrupados en cinco dimensiones, tal y como se refleja en la tabla 1 . 


\section{Tabla 1: Modelo Compround}

\begin{tabular}{|c|c|}
\hline Dimensión & Indicador \\
\hline \multirow{9}{*}{$\begin{array}{l}\text { Diseño de los } \\
\text { programas/ } \\
\text { guías docentes } \\
\text { de la asignatura }\end{array}$} & 1. Vinculación de los contenidos con los diferentes aspectos de la profesión \\
\hline & 2. Adecuación de las competencias con el desarrollo de la profesión \\
\hline & 3. Adecuación de los objetivos con el perfil profesional de la titulación \\
\hline & 4. Utilidad de las prácticas para el desarrollo de competencias profesionales \\
\hline & 5. Adecuación de la metodología de enseñanza a las formas de aprendizaje del alumnado \\
\hline & 6. Adecuación de las prácticas al contexto teórico de la asignatura \\
\hline & 7. Adecuación de los sistemas de evaluación empleados con las diferentes tareas propuestas \\
\hline & 8. Adecuación del número de prácticas al desarrollo de la asignatura \\
\hline & $\begin{array}{l}\text { 9. Existencia de sistemas de coordinación de las actividades teóricas y prácticas previstas en el } \\
\text { programa }\end{array}$ \\
\hline \multirow{16}{*}{$\begin{array}{l}\text { Metodología } \\
\text { docente }\end{array}$} & 10. Fomento de la participación en clase y motivación del alumnado \\
\hline & 11. Uso de metodologías para implicar activamente a los estudiantes en el aprendizaje \\
\hline & 12. Seguimiento del proceso de aprendizaje del alumnado \\
\hline & 13. Desarrollo de la capacidad de síntesis y de razonamiento del alumnado \\
\hline & 14. Promoción del desarrollo en el alumno de una actitud reflexiva \\
\hline & 15. Creación de un clima de trabajo y participación \\
\hline & 16. Estímulo al alumnado para que se interese por su proceso de aprendizaje \\
\hline & $\begin{array}{l}\text { 17. Reparar en la opinión de los estudiantes en cuestiones relacionadas con el desarrollo de la } \\
\text { docencia }\end{array}$ \\
\hline & $\begin{array}{l}\text { 18. Propuesta de actividades para favorecer el aprendizaje autónomo (búsqueda de información } \\
\text { complementaria, trabajos, investigaciones, etc.) }\end{array}$ \\
\hline & 19. Variedad de procedimientos para evaluar el aprendizaje del alumnado \\
\hline & 20. Elaboración de manuales didácticos de apoyo al desarrollo de la asignatura \\
\hline & 21. Diseño de cuadernos de prácticas para su implementación en el aula \\
\hline & 22. Evaluación coherente con los objetivos, contenidos, metodología y actividades del curso \\
\hline & $\begin{array}{l}\text { 23. Exposición de ejemplos en los que se ponen en práctica las competencias a desarrollar por la } \\
\text { asignatura }\end{array}$ \\
\hline & 24. Coherencia entre los objetivos planificados y las tareas de enseñanza \\
\hline & 25. Presentación de los conceptos básicos de la material a nivel teórico y práctico \\
\hline \multirow{7}{*}{$\begin{array}{l}\text { Coherencia } \\
\text { interna de los } \\
\text { recursos } \\
\text { didácticos }\end{array}$} & 26. Aporte de bibliografía y/o fuentes de información relevantes \\
\hline & 27. Adecuación de la bibliografía y los recursos con las finalidades de la asignatura \\
\hline & 28. Relación entre los distintos bloques temáticos de la materia \\
\hline & $\begin{array}{l}\text { 29. Organización y presentación de los materiales adecuada (explicaciones, materiales escritos, } \\
\text { actividades, etc.) }\end{array}$ \\
\hline & 30. Elaboración de manuales didácticos de apoyo al desarrollo de la asignatura \\
\hline & 31. Diseño de cuadernos de prácticas para su implementación en el aula \\
\hline & $\begin{array}{l}\text { 32. Coordinación entre el profesorado para evitar solapamientos y relacionar los contenidos de } \\
\text { las distintas asignaturas }\end{array}$ \\
\hline \multirow{3}{*}{$\begin{array}{l}\text { Información } \\
\text { sobre sistemas } \\
\text { de evaluación }\end{array}$} & 33. Información del sistema de evaluación al finalizar el curso \\
\hline & 34. Información del sistema de evaluación durante el transcurso de la docencia \\
\hline & 35. Información del sistema de evaluación al comienzo del curso \\
\hline \multirow{6}{*}{$\begin{array}{l}\text { Actitud del } \\
\text { profesorado }\end{array}$} & 36. Respeto hacia el alumnado \\
\hline & 37. Interés por la asignatura por parte del docente \\
\hline & 38. Actitud receptiva en su relación con el alumnado \\
\hline & 39. Promoción de una comunicación fluida y espontánea \\
\hline & 40. Atención ante las dificultades de aprendizaje de sus estudiantes \\
\hline & 41. Interés por las experiencias y las representaciones del alumnado \\
\hline
\end{tabular}


Dicho modelo fue posteriormente validado por parte del personal del laboratorio LIFE (Universidad de Ginebra, Suiza), institución europea destinada a la formación y evaluación de competencias docentes universitarias, lo que aportó al mismo una perspectiva de transferencia internacional para todas aquellas instituciones universitarias que velan por la calidad de sus profesionales.

Una aproximación a los elementos que incidirán en la configuración de un profesor o profesora universitaria de calidad y, en definitiva, claves para la evaluación de su actividad como docente y que definen el modelo Compround, son (López, González y León, 2015):

- Metodología docente: la metodología se entiende como un conjunto coherente de técnicas y acciones, lógicamente coordinadas para dirigir el aprendizaje del alumnado hacia determinados objetivos, dando por tanto respuesta a la pregunta cómo enseñar; en este sentido, el profesorado ha de tomar una serie de decisiones sobre qué métodos emplear en el aula (individualizados, socializadores, cooperativos, etc.), qué estrategias de enseñanza y aprendizaje va a poner en marcha, cuál va a ser la organización espacial, cómo va a distribuir el tiempo, qué tipo de relaciones sociales y agrupamientos va a generar con su grupo de alumnos y alumnas, qué tipo de actividades va a proponer y cuál va a ser su secuencia, cómo atenderá a la diversidad del alumnado, etc.

- Diseño de las guías docentes de la asignatura: en los tradicionales programas de las materias el núcleo rebotaba sobre el contenido, sin embargo, en el caso de las nuevas guías docentes donde la acción formativa está basada en competencias, el centro de atención es doble: el contenido y el trabajo del estudiante en torno al mismo. Desde esta perspectiva se entiende que la docencia no solo se puede limitar a la actividad en el aula sino que habrá que extenderla a otros escenarios así como promover actividades prácticas llevadas a cabo, tanto por el profesorado, como por el alumnado, con el objetivo de desarrollar las competencias profesionales a través de la contextualización de los contenidos teóricos y de su transferencia a la vida cotidiana. Estas actividades se definen como acciones de interacción y participación activa y conjunta, con la intención de identificar, orientar, apoyar, fomentar y promover en las comunidades el proceso de formación del alumnado.

- Actitud del profesorado: la actitud del profesorado puede considerarse como el estado de preparación" o disposición mental o psicológica y neutral, que se organiza a través de la experiencia y que influye en la respuesta del sujeto (Allport, 1935, p. 830). Desde esta perspectiva, es imprescindible que un buen o una buena docente promocione momentos de comunicación bidireccional y preste interés a las expectativas e intereses de su alumnado.

- Coherencia interna de los recursos didácticos: Los recursos didácticos son todos aquellos medios empleados por el profesorado en el diseño o desarrollo del currículum (por su parte o por la del alumnado) para aproximar o facilitar los contenidos, mediar en las experiencias de aprendizaje, provocar encuentros o situaciones, desarrollar habilidades cognitivas, apoyar sus estrategias metodológicas o facilitar o enriquecer la evaluación (Blázquez y Lucero, 2002). En este sentido, el buen o buena docente ha de ser capaz de localizar, organizar, emplear y adaptar los distintos recursos a los distintos momentos de su labor profesional. 
- Información sobre sistemas de evaluación: la información que el o la docente aporta al alumnado sobre los sistemas de evaluación empleados se puede considerar como el referente evaluativo en el que se integraran los criterios de evaluación y calificación, las técnicas e instrumentos de evaluación, así como los momentos en los que la evaluación sería efectuada.

En resumen, el perfil docente derivado de este modelo responde a un profesional que desarrolla su actividad sobre la base de una labor planificada de forma sistemática y con suficiente antelación, donde la información a transmitir está ligada directamente con las competencias profesionales del trabajo para el que capacita la titulación en la que se encuentra y que emplea recursos metodologías y estrategias didácticas en un contexto específico de enseñanza-aprendizaje donde la planificación docente, la gestión del tiempo y del espacio, y los sistema de evaluación y la interacción con el alumnado juegan un papel importante (López, González y León, 2014: 146).

\subsection{LA EVALUACIÓN DOCENTE EN LA UNIVERSIDAD DE CONCEPCIÓN (CHILE)}

Las universidades chilenas cuentan con sistemas de evaluación del trabajo de sus académicos vinculados a la investigación, a la docencia y a la extensión, con influencia directa, en su mayoría, en la remuneración de este grupo de profesionales. Tal y como relatan Montoya, Arbesú, Contreras y Conzuelo (2014), el área de docencia suele aparecer valorada a partir de criterios tales como la cantidad de cursos dictados en un período académico, si son de pre o posgrado; la elaboración de material didáctico; la atención de alumnado; la dirección de tesis y seminarios, y las supervisiones de prácticas. Estos criterios son, en la mayoría de los casos, cuantitativos y sobre la base prioritaria de la opinión del alumnado.

En el caso de la Universidad de Concepción (UdeC), la docencia es entendida como una actividad fundamental y prioritaria ya que está implementando una oferta formativa flexible y abierta con opciones articuladas y basadas en resultados de aprendizaje y orientada al desarrollo de competencias. Se desprende, del modelo educativo de esta institución (Universidad de Concepción, 2011), que los y las estudiantes serán partícipes de las innovaciones y de los cambios orientados a formarlos como profesionales de excelencia, creativos, reflexivos, críticos y sensibles a los problemas de la sociedad.

Es por ello que la forma de entender los procesos de enseñanza-aprendizaje involucra cambios en los procesos formativos y se adscribe a una concepción curricular orientada al desarrollo de competencias, nuevas formas de enseñar y aprender, nuevas metodologías para enfrentar la diversidad de estilos de aprendizaje, nuevas formas de evaluar los resultados de aprendizaje, la inserción de las Tecnologías de Información y Comunicación en la enseñanza, el aprendizaje y la gestión del conocimiento (Universidad de Concepción, 2011: 7).

El trabajo realizado por Aguilar, Ampuero y Loncomilla (2007), al respecto de la opinión manifestada por el alumnado de doce universidades chilenas pertenecientes al Consejo de Rectores de Universidades Chilenas (CRUCH) confirmó que, si bien valoraron la existencia de un mecanismo que permitiese evaluar al profesorado o al proceso docente, señalaron que los instrumentos utilizados son poco eficientes debido a la forma de implementación (en un $81 \%$ vía on-line), a los períodos escogidos (una semana al final de cada semestre), a la excesiva rigidez de las cuestiones planteadas y a la poca apertura de las mismas para aportar opiniones divergentes, así como el nulo efecto que los resultados tienen en el sistema universitario, entendiéndose en exclusiva como un sistema de rendición de cuentas administrativo. 
En el polo opuesto, nos encontramos con un $52 \%$ de estudiantes que opinaron que las herramientas existentes en sus universidades estaban bien formuladas, incorporando todos los elementos que, desde su percepción, permiten evaluar a un docente, y se entendieron como una buena forma para que el profesorado tenga conocimiento sobre sus fortalezas y debilidades.

¿Y la opinión manifestada por el profesorado de estas mismas universidades? Báez, Cazenave y Lagos (2007) analizaron, entre otros, las implicaciones de los resultados de la evaluación del desempeño docente entrevistando a 64 profesores y profesoras de 11 universidades chilenas. Evidenciaron que un $33 \%$ consideró la no existencia de cambios, impacto o efectos correctivos, a su vez que no existía retroalimentación colegiada ni implicaciones institucionales por el nivel de desempeño logrado. Sin embargo, un $67 \%$ encontró algunas implicaciones significativas que podían atender a diferentes elementos de su labor profesional.

La tabla 2 muestra las diferentes opiniones vertidas por este grupo de profesoras y profesores a partir del peso reportado por sus respuestas, que los autores de este artículo hemos agrupado en tres ámbitos (planificación de la docencia, efectos contractuales y desarrollo profesional) y que hay que considerar como referentes en la definición de modelos de evaluación de la calidad de la actividad docente.

Tabla 2: Implicaciones de la evaluación de la actividad docente

\begin{tabular}{|c|c|c|}
\hline $\begin{array}{l}\text { Planificación de la docencia } \\
\qquad(57 \%)\end{array}$ & $\begin{array}{l}\text { Efectos contractuales } \\
\qquad(28 \%)\end{array}$ & $\begin{array}{l}\text { Desarrollo profe } \\
\qquad(27 \%)\end{array}$ \\
\hline $\begin{array}{l}\text { Permite la retroalimentación } \\
\text { (fortalezas y debilidades) para } \\
\text { corregir y mejorar la gestión } \\
\text { docente/ Calidad de la } \\
\text { enseñanza. }\end{array}$ & $\begin{array}{l}\text { Permite definir la permanencia } \\
\text { en el cargo de profesores de } \\
\text { tiempo parcial o sus } \\
\text { posibilidades de renovación de } \\
\text { contrato. }\end{array}$ & $\begin{array}{l}\text { Permite evaluar publicacion } \\
\text { y compromiso académico. }\end{array}$ \\
\hline $\begin{array}{l}\text { Capacitación a docentes mal } \\
\text { evaluados }\end{array}$ & $\begin{array}{l}\text { En profesores a tiempo } \\
\text { completo se usa para su } \\
\text { calificación y podría ser motivo } \\
\text { de despido si sus calificaciones } \\
\text { bajas son reiterativas. }\end{array}$ & $\begin{array}{l}\text { Estímulo de reconocimiento } \\
\text { para el profesorado bien } \\
\text { evaluado que implica mejoras } \\
\text { salariales. }\end{array}$ \\
\hline $\begin{array}{l}\text { Permite corregir metodologías } \\
\text { de trabajo en clases y/o } \\
\text { laboratorios, reducir o aumentar } \\
\text { tamaño de las sesiones, cambios } \\
\text { de horario, cambios de plan de } \\
\text { compra de materiales, etc. }\end{array}$ & & $\begin{array}{l}\text { Ayuda a las autoridades } \\
\text { académicas a focalizar los } \\
\text { compromisos para el siguiente } \\
\text { período docente, facilitando la } \\
\text { toma de decisiones. }\end{array}$ \\
\hline
\end{tabular}

Fuente: elaboración propia a partir de Báez, Cazenave y Lagos (2007)

La función docente de los académicos de la UdeC es clave para el cumplimiento de la misión institucional. Dentro de este contexto, se plantea un perfil docente como una visión que debe poseer todo el profesorado y donde la universidad ha de aportar todos los medios y oportunidades necesarias para alcanzarlo. Este está constituido por una serie de ámbitos de desempeño, cada uno de los cuáles queda definido por una serie de competencias, tanto genéricas como específicas (ver tabla 3). 
Tabla 3: Perfil docente de la UdeC

\begin{tabular}{lll}
\hline \multicolumn{1}{c}{$\begin{array}{c}\text { Ámbitos de } \\
\text { desempeño }\end{array}$} & \multicolumn{1}{c}{ Competencias } & Competencias específicas \\
\hline & $\begin{array}{l}\text { C1. Demostrar dominio del } \\
\text { cuerpo de conocimientos } \\
\text { relativos a su disciplina y la }\end{array}$ & $\begin{array}{l}\text { C1.1 Demostrar conocimiento disciplinar sólido, a través de la } \\
\text { docencia }\end{array}$ \\
A1. Conocimiento \\
reconstrucción del mismo, \\
disciplinar
\end{tabular}
en una segunda lengua.

C2.1 Definir resultados de aprendizaje coherentes con las competencias del perfil de egreso establecidas en el plan de estudios.

C2.2. Organizar y preparar los contenidos disciplinares, velando por la significatividad de los aprendizajes.

C2.3 Diseñar estrategias pedagógicas para favorecer el logro de los aprendizajes, considerando el establecimiento de conexiones con los aprendizajes previos y con los previstos en los niveles superiores

A2. Gestión del proceso de enseñanza y aprendizaje
C2. Planificar el proceso de del plan de estudios.

enseñanza - aprendizaje, C2.4 Favorecer situaciones de comunicación y relación con los considerando los resultados estudiantes que propician la motivación, las altas expectativas y con de aprendiza- je ello, el aprendizaje.

comprometidos para los $\mathrm{C} 2.5$ Organizar el tiempo, el espacio físico y los recursos estudiantes.

\section{disponibles, optimizando las condiciones adecuadas para el} desarrollo de las tareas de aprendizaje.

C2.6 Diseñar actividades coherentes con los resultados de aprendizaje y el tiempo disponible de los estudiantes, tanto para actividades supervisadas como no supervisadas (SCT).

C2.7 Incorporar las TIC como medio eficaz de interacción pedagógica, superando su dimensión meramente instrumental.

C2.8 Retroalimentar y apoyar oportunamente al estudiante en su desarrollo académico y en su formación integral.

C3.1 Diseñar diversos instrumentos de evaluación que permitan recoger información y retroalimentar el proceso formativo de los estudiantes.

A3. Gestión en el Ámbito de la Evaluación para la Autorregulación de los Aprendizajes
C3. Evaluar el proceso de enseñanza - aprendizaje a través de diversos procedimientos instrumentos.

C3.2 Comunicar los criterios y los resultados del proceso evaluativo que permitan al estudiante ser participante reflexivo de su propio proceso de aprendizaje.

C3.3 Monitorear sistemáticamente los resultados del proceso evaluativo.

C3.4 Tomar decisiones de los resultados del proceso de enseñanza y aprendizaje en función de los resultados del proceso evaluativo.

C4.1 Autoevaluar su propio desempeño docente, con actitud de apertura al aprendizaje y a la mejora continua.

C4.2 Reflexionar acerca de sus prácticas pedagógicas incorporando

A4. Gestión en el

Ámbito del

Desarrollo

Profesional para la

Mejora de las

Prácticas Docentes
C4. Generar instancias a otros actores (estudiantes, pares, egresados, otros).

sistemáticas de reflexión C4.3 Diseñar, implementar y evaluar proyectos de apoyo a la orientadas a mejorar e docencia asociados a problemáticas propias de su práctica docente. innovar en la práctica C4.4 Trabajar en equipos y mostrar disposición a identificarse con docente la misión institucional y su proyecto educativo.

C4.5 Coordinar actividades de aprendizaje con otros profesionales internos o externos para fortalecer el aprendizaje integral de los estudiantes, aprovechando los recursos institucionales. 
Estudios Pedagógicos XLII, Nº 4: 69-85, 2016

CLAVES DE COMPROUND PARA LA REDEFINICIÓN DEL MODELO DE EVALUACIÓN DE LA CALIDAD DOCENTE EN LA UNIVERSIDAD DE CONCEPCIÓN

Este modelo de docente se define mediante cuatro ámbitos de desempeño que dan lugar a sendas competencias que se subdividen, a su vez, en un total de 19 competencias específicas. El mismo refleja una figura docente basada en aquel profesional con un alto dominio cognitivo y epistemológico de las materias que imparte, siendo capaz de transmitirlas en un acto didáctico programado y adaptado al alumnado que recibe sus enseñanzas, así como evaluarlas de acuerdo a unos criterios fiables y válidos que permitan el desarrollo académico de sus estudiantes, todo ello en un contexto de trabajo colaborativo e interdisciplinar.

Es interesante percibir que este modelo entronca directamente con la herramienta que establece la universidad para evaluar la calidad de la labor profesional de su profesorado, a valorar desde la perspectiva del alumnado. Esta se constituye como un cuestionario de valoración individual por asignatura y profesorado denominado Encuesta de Evaluación Docente y formada por 14 ítems que reflejan sendas competencias, a responder a lo largo de una escala de cuatro puntos que abarcan la valoración de su consecución desde nunca a siempre, a las que se añade una pregunta de respuesta libre cuyo objetivo es comentar aquellos aspectos que se consideran positivos así como aquellos que debieran mejorarse. La cumplimentación de la misma se realiza de manera autoadministrada sin presencia del encuestador, a través de una plataforma virtual.

Los elementos de valoración por parte de las y los estudiantes en referencia a su profesorado que se desvelan en este instrumento son los siguientes:

1. Conocimiento disciplinar sólido a través de la docencia, demostración a los y las estudiantes su interés por la materia que imparte y respuestas precisas a las preguntas del alumnado.

2. Ajuste al plan de trabajo previsto en la planificación y coordinación con las actividades de las clases prácticas, laboratorios o clínicas.

3. Fomento del aprendizaje independiente en los estudiantes y ofrecimiento de diferentes puntos de vista sobre un mismo tema.

4. Relación de los contenidos con los temas que ya conoce el alumnado, establecimiento de relaciones con otros conocimientos y experiencias, y empleo de ejemplos para ilustrar los contenidos.

5. Establecimiento claro de los criterios para evaluar los conocimientos de los alumnos y las alumnas y comunicación oportuna de los mismos.

6. Evaluación del alumnado de acuerdo con los resultados de aprendizaje establecidos en la planificación del curso.

7. Adaptación de la evaluación con el nivel de exigencia impartido.

8. Revisión de acuerdo a la pauta establecida en la planificación de la asignatura.

9. Cumplimiento de los horarios de clase, clínicas, atención de alumnos y otras actividades establecidas.

10. Entrega a través de la plataforma INFODA de los resultados de las evaluaciones dentro de los plazos reglamentarios.

11. Disposición de los materiales de apoyo al aprendizaje.

12. Mantenimiento de una relación cordial y un trato respetuoso con el alumnado y otros miembros de la comunidad universitaria.

13. Satisfacción general con el profesor o profesora.

14. Asistencia a las clases.

Esta taxonomía evoca una serie de dimensiones que permiten definir el perfil de profesorado que se desea para el alumnado de la UdeC así como para tener en cuenta en un sistema de evaluación de la calidad de la docencia, y que son: dominio de la materia 
a impartir, planificación didáctica de la materia, evaluación de los aprendizajes del alumnado, gestión administrativa de las labores docentes y actitudes. Estas dimensiones están presentes en el modelo docente ya establecido el año 2015 por la universidad.

Al finalizar el proceso, el profesorado recibe un informe de evaluación que incorpora el porcentaje de respuesta de valoración del conjunto de estudiantes a cada competencia sometida a juicio, así como las respuestas libres aportadas por el alumnado sobre debilidades y fortalezas del trabajo ejercido por su profesorado.

Se concibe, por tanto, como una evaluación formativa que busca conocer las fortalezas y debilidades en cada aspecto de la docencia, con la finalidad de ayudar al profesorado a mejorar su trabajo diario y contribuir de esta manera a su desarrollo profesional (Stake, Contreras y Arbesú, 2011). Sin embargo, la evaluación que realmente se reconoce es la sumativa, en tanto que busca determinar la calidad de la docencia y asegurar dicha calidad, con efectos directos sobre el espectro contractual del profesorado basado en premios, reconocimientos y sanciones.

\section{MÉTODO}

Fue nuestra intención conocer la opinión del profesorado de la Universidad de Concepción acerca del procedimiento evaluativo al que se ven sometidos como profesionales de la docencia y establecer la aportaciones que el modelo Compround puede aportar a la generación de un perfil competencial docente que sirva de base para optimizar los procesos de evaluación de la calidad del profesorado.

Para lograr este propósito implementamos un grupo de discusión, ya que es un instrumento que posee ventajas únicas a la hora de ofrecer información en profundidad sobre las necesidades, intereses y preocupaciones de un colectivo social determinado (Krueger, 1991), en este caso 12 docentes procedentes de las áreas de Educación Física, Pedagogía Diferencial y Pedagogía Matemática de la UdeC. Este grupo de profesionales estaba caracterizado por su experiencia docente (más de cinco años), su carácter interdisciplinar (imparten docencia en diferentes titulaciones) y su alto interés por la innovación educativa. Fueron dos las preguntas estímulo que se realizaron: valoración del proceso de evaluación de la labor docente por parte del alumnado y efectos que la misma tiene sobre su trabajo.

Asimismo, se llevó a cabo un estudio documental-comparativo (Del Rincón et al., 1995) entre los modelos Compround y el desarrollado por la UdeC, con el fin de obtener información útil y necesaria a través del análisis de documentos escritos para dar respuesta a la meta planteada en este trabajo.

\section{RESULTADOS OBTENIDOS}

\subsection{RESULTADOS DEL GRUPO FOCAL}

Un análisis general de las respuestas ofrecidas por los y las participantes del grupo de discusión indicó que la mayoría de las opiniones vertidas fueron de carácter negativo y se centraron básicamente en la falta de control del sistema, la escasa información reportada y la baja repercusión que la misma tiene sobre su trabajo. 
Si hacemos referencia a la primera de las cuestiones planteadas, valoración del sistema de evaluación docente, las aportaciones más significativas se refieren a la subjetividad del sistema, a la escasez de información que aporta como recurso formativo para el profesorado y a la falta de implicación del alumnado como emisor de la información. Las respuestas literales más recurrentes se resumen en las siguientes:

- Arbitrariedad en las respuestas por parte del alumnado.

- Falta de control sobre el proceso de cumplimentación por parte del alumnado, al ser esta cumplimentada a través de internet sin la presencia de ningún encuestador o encuestadora, evidenciando una baja tasa de respuestas.

- El informe de resultados se centra únicamente en la frecuencia de respuestas dadas por el alumnado a cada unidad de valoración escalar dentro del rango y no ofrece valores globales.

- Hay profesorado que no sabe interpretar los datos obtenidos.

- Opinión subjetiva del alumnado sobre la docencia, donde influyen elementos tales como la simpatía al profesor, momento del año en el que se aplica, el tipo de curso, carrera que cursa el alumnado, evaluación realizada por alumnado que no asiste con regularidad a las clases, etc.

Si nos centramos en el segundo de los núcleos de la discusión, los efectos de la evaluación en su trabajo docente, la mayoría señaló que son nulos los efectos administrativos de los resultados obtenidos y que el alumnado utiliza este procedimiento de manera punitiva, al objeto de "castigar" al profesorado. A pesar de ello, hubo algunas voces que indicaron la utilidad del sistema en el sentido de que el informe de resultados que reciben aporta información orientativa que nos permite reconfigurar la docencia para el curso siguiente, siempre con el apoyo de otras fuentes de información que podrían implementarse tales como autoevaluaciones personales y reuniones con compañeros de trabajo que puedan interpretarse como una evaluación entre iguales.

\subsection{RESULTADOS DEL ESTUDIO DOCUMENTAL-COMPARATIVO}

La UdeC clasifica las competencias de su modelo docente en cuatro ámbitos de desempeño, mientras que el modelo Compround establece cinco dimensiones competenciales. Por su parte, el primero de los modelos define un perfil formado por 19 competencias mientras que el segundo engloba un total de 41. La lectura de estos datos así como la información aportada en la tabla 4, evidencia una diferencia significativa entre ambos escenarios pero que comparten elementos comunes que convierten ambos perfiles en modelos válidos y científicamente aceptados. 
Tabla 4: Comparación modelo UdeC y Modelo Compround

\begin{tabular}{|c|c|c|c|}
\hline \multicolumn{2}{|l|}{ Compround } & \multicolumn{2}{|l|}{ UdeC } \\
\hline Dimensiones & Indicadores & Ámbitos de desempeño & Competencias \\
\hline $\begin{array}{l}\text { 1. Diseño de los programas/ } \\
\text { guías docentes de la asignatura }\end{array}$ & 9 & 1. Conocimiento disciplinar & 2 \\
\hline 2. Metodología docente & 16 & $\begin{array}{l}\text { 2. Gestión del proceso de } \\
\text { enseñanza y aprendizaje }\end{array}$ & 8 \\
\hline 3. Recursos didácticos & 7 & - & - \\
\hline 4. Sistemas de evaluación & 3 & $\begin{array}{l}\text { 3. Gestión en el Ámbito de la } \\
\text { Evaluación para la Autorregulación } \\
\text { de los Aprendizajes }\end{array}$ & 4 \\
\hline \multirow[t]{2}{*}{ 5. Actitud del profesorado } & 6 & - & - \\
\hline & - & $\begin{array}{l}\text { 4. Gestión en el Ámbito del } \\
\text { Desarrollo Profesional para la } \\
\text { Mejora de las Prácticas Docentes }\end{array}$ & 5 \\
\hline Total & 41 & Total & 19 \\
\hline
\end{tabular}

Es interesante señalar la existencia de ámbitos de desempeño o dimensiones competenciales comunes, tales como las referidas al conocimiento disciplinar (diseño y actualización de la materia), a la gestión del proceso de enseñanza y aprendizaje (metodología docente empleada en el aula) y la gestión de los sistemas de evaluación del alumnado. A nivel diferencial, encontramos que el modelo UdeC incorpora un ámbito no presente en Compround y que se identifica como la Gestión del Desarrollo Profesional (innovación docente), mientras que este último incorpora dimensiones referidas a los recursos didácticos y a la actitud del profesorado.

A partir de una lectura y análisis sistemático del contenido de ambos modelos, se ha procedido a realizar una combinación de ambos en el sentido de aportar una visión única al amparo de los parámetros establecidos por la UdeC y los deseos y expectativas marcadas por el profesorado participante en el grupo de discusión. Se ha procurado utilizar una redacción de consenso de las diferentes dimensiones y elementos competenciales a partir de la nomenclatura empleada por la UdeC y hemos tratado de eliminar elementos repetidos, reubicando componentes entre las dimensiones extraídas, unificando competencias similares y redenominando otras.

Se ha conseguido elaborar un modelo teórico formado por un total de seis ámbitos de desempeño (dimensiones de evaluación competencial) y 37 competencias o indicadores de evaluación que definen un perfil docente muy concreto, tal y como se detalla en la tabla 5 . 
Tabla 5: Propuesta del nuevo perfil docente para la UdeC

\begin{tabular}{l}
\hline $\begin{array}{l}\text { Ámbitos de } \\
\text { desempeño }\end{array}$ \\
\hline \\
1. Conocimiento \\
disciplinar
\end{tabular}

disciplinar

Competencias

1. Demostrar conocimiento disciplinar sólido.

2. Vincular los contenidos con los diferentes aspectos de la profesión.

3. Adecuar las competencias con el desarrollo de la profesión.

4. Adecuar los objetivos con el perfil profesional de la titulación.

5. Adecuar la metodología de enseñanza a las formas de aprendizaje del alumnado.

6. Adecuar las prácticas al contexto teórico de la asignatura.

7. Adecuar los sistemas de evaluación empleados con las diferentes tareas propuestas.

8. Adecuar el número de prácticas al desarrollo de la asignatura.

9. Diseñar de sistemas de coordinación de las actividades teóricas y prácticas.

10. Definir resultados de aprendizaje coherentes con las competencias del perfil de egreso establecidas en el plan de estudios.

11. Organizar y preparar los contenidos disciplinares, velando por la significatividad de los aprendizajes.

12. Diseñar estrategias pedagógicas que favorezcan el logro de los aprendizajes, considerando el establecimiento de conexiones con los aprendizajes previos y con los previstos en los niveles superiores del plan de estudios.

2. Gestión del proceso de enseñanza y aprendizaje

13. Favorecer situaciones de comunicación y relación con los estudiantes que propicien la participación activa y la motivación.

14. Organizar el tiempo, el espacio físico y los recursos disponibles, optimizando las condiciones adecuadas para el desarrollo de las tareas de aprendizaje.

15. Diseñar actividades coherentes con los resultados de aprendizaje y el tiempo disponible de los estudiantes, tanto para actividades supervisadas como no supervisadas (SCT).

16. Retroalimentar al estudiante en su desarrollo académico y en su formación integral.

17. Estimular al alumnado para que se interese por su proceso de aprendizaje

18. Reparar en la opinión de los estudiantes en cuestiones relacionadas con el desarrollo de la docencia

19. Diseñar instrumentos de evaluación que permitan recoger información y retroalimentar el proceso formativo de los estudiantes.

3. Gestión en el ámbito de la evaluación de los aprendizajes del alumnado

20. Comunicar los criterios y los resultados del proceso evaluativo que permitan al estudiante ser participante reflexivo de su propio proceso de aprendizaje, al comienzo, durante y al finalizar el curso académico.

21. Informar sistemáticamente al alumnado sobre los resultados del proceso evaluativo.

22. Tomar decisiones sobre el proceso de enseñanza y aprendizaje en función de los resultados del proceso evaluativo.

23. Autoevaluar el propio desempeño docente, con actitud de apertura al aprendizaje y a la mejora continua.

24. Reflexionar acerca de las prácticas pedagógicas incorporando a otros actores

4. Gestión en el ámbito de la innovación docente (estudiantes, pares, egresados, etc.).

25. Diseñar, implementar y evaluar proyectos de apoyo a la docencia asociados a problemáticas propias de su práctica docente.

26. Trabajar en equipo y mostrar disposición a identificarse con la misión institucional y su proyecto educativo.

27. Coordinar actividades de aprendizaje con otros profesionales internos $o$ externos para fortalecer el aprendizaje integral del alumnado. 


\begin{tabular}{|c|c|}
\hline \multirow{5}{*}{$\begin{array}{l}\text { 5. Recursos } \\
\text { didácticos }\end{array}$} & 28. Aportar bibliografía y/o fuentes de información relevantes. \\
\hline & 29. Adecuar la bibliografía y los recursos con las finalidades de la asignatura. \\
\hline & 30. Organizar y presentar los materiales de manera adecuada. \\
\hline & 31. Elaborar manuales didácticos de apoyo al desarrollo de la asignatura. \\
\hline & 32. Diseñar cuadernos de prácticas para su implementación en el aula. \\
\hline \multirow{5}{*}{$\begin{array}{l}\text { 6. Actitud del } \\
\text { profesorado }\end{array}$} & 33. Respeto hacia el alumnado. \\
\hline & 34. Interés por la asignatura por parte del docente. \\
\hline & 35. Actitud receptiva en su relación con el alumnado. \\
\hline & 36. Atención ante las dificultades de aprendizaje de los estudiantes. \\
\hline & 37. Interés por las experiencias y las representaciones del alumnado. \\
\hline
\end{tabular}

De este modelo se define un perfil docente que, ya definido anteriormente como un profesional que domina cognitivamente las materias y transmite en un acto didáctico sistemáticamente programado, siendo capaz de evaluar a su alumnado desde una perspectiva tanto formativa como sumativa, incorpora al perfil la capacidad para el diseño de recursos didácticos de apoyo a su labor docente y la posesión de una actitud que favorece la implicación activa del alumnado en su proceso de aprendizaje.

La propuesta aquí diseñada permite validar el estudio realizado por Casero (2008), que estableció como dimensiones de evaluación de la actividad docente las siguientes: cumplimiento de las obligaciones, programa, conocimiento/interrelación con la materia, metodología, evaluación y satisfacción. Sin embargo, la futura realización de una análisis factorial confirmatorio permitirá validar la estructura contextual resultante de la exploración aquí realizada.

Se trata, pues, de una propuesta activa y útil desde la perspectiva del alumnado que no debe hacernos perder de vista la necesidad de seguir avanzando en pro de la excelencia académica y todo lo que conlleva su significado para la Universidad de Concepción.

\section{4. ÚLTIMOS COMENTARIOS}

Independientemente del tipo de evaluación realizada, se concibe como algo positivo el disponer de un sistema de evaluación de la calidad de la labor docente del profesorado en tanto supone un proceso de retroalimentación de su labor profesional y una oportunidad del destinatario de su trabajo, el alumnado, de aportar una visión de la labor desarrollada por su profesorado.

Dado que el objetivo de esta evaluación es la mejora y el perfeccionamiento de las actuaciones ligadas con la docencia, sería interesante incorporar al proceso de evaluación de la docencia nuevos procedimientos nombrados por el profesorado que permitan triangular la percepción manifestada por el alumnado y contar, de este modo, con otros focos de interés. Por un lado, sería recomendable llevar a cabo labores de autoevaluación mediante autoinformes docentes que recojan evidencias de la labor realizada por parte del protagonista de los procesos de enseñanza, informes de evaluación de gestores académicos encargados de planificar y organizar la labor docente del profesorado, y sistemas de evaluación por pares, entendida como una forma específica de aprendizaje colaborativo en el que los participantes realizan una valoración conjunta sobre la labor docente ejercida. Se puede 
considerar que este tipo de evaluación, tal y como señalan Prins, Sluijsmans, Kirschner y Strijbos (2005), es una de las formas más efectivas para promover la colaboración y cooperación entre el profesorado.

Asimismo, se sugiere que la UdeC promueva acciones que refuercen y estimulen las buenas prácticas de aula y que permitan erradicar y evitar aquellas menos eficaces, aportando de este modo nuevas evidencias que definan la calidad de su profesorado. Es por ello que abogamos por la convocatoria de proyectos de innovación docente liderados por el profesorado, con el propósito de promover propuestas innovadoras que redunden en la mejora de los procesos de enseñanza-aprendizaje y en los resultados académicos del alumnado, así como actividades de formación continua dirigidas a paliar las debilidades formativas del profesorado, a formar en nuevos procesos pedagógicos y didácticos, y a optimizar las buenas prácticas docentes.

En definitiva, la evaluación integral de la labor docente del profesorado universitario debería orientarse, tal y como expresan Jornet, González y Sánchez (2014: 187), a través de modelos globales, sistémicos, en los que los elementos que concurren en la acción individual permitieran contextualizar de manera adecuada la actuación individual. Por ello, la evaluación del profesorado debe enmarcarse en un proceso genérico de calidad institucional y de los sistemas educativos dirigido a la evaluación de la docencia. No se trata, pues, de posturas antagónicas, sino necesariamente complementarias si lo que se pretende es abordar la evaluación del profesorado desde una perspectiva de validez que represente sus facetas fundamentales: credibilidad, utilidad, justicia y equidad.

Bajo todos estos parámetros, podemos concluir que la evaluación de la actividad docente del profesorado no debe basarse únicamente en el reconocimiento mediante la demostración de una serie de competencias, sino que la docencia deber consistir en un proceso de intervención que atienda tanto a la planificación del proceso de enseñanza como al desempeño o desarrollo, a los resultados y a las estrategias puestas en marcha en torno a la innovación y la mejora (Universidad de Córdoba, 2010).

\section{REFERENCIAS BIBLIOGRÁFICAS}

Aguilar, A.; Ampuero, N. y Loncomilla, L. (2007). Evaluación del desempeño docente. Diagnóstico desde la perspectiva de los estudiantes. En CINDA. Evaluación del desempeño docente y calidad de la docencia universitaria (pp. 86-97). Santiago de Chile: CINDA.

Allport, G.W. (1935). Attitudes. En C. Murchison (Ed.). A Handbook of Social Psychology (pp. 798844). Worchester (Massachusetts): Clark University Press.

Báez, M.; Cazenave, M. y Lagos, J. (2007). Percepción de la evaluación del desempeño docente: diagnóstico desde la perspectiva del docente. En CINDA. Evaluación del desempeño docente y calidad de la docencia universitaria (pp. 57-85). Santiago de Chile: CINDA.

Blázquez, F. y Lucero, M. (2002). Los medios y recursos en el proceso didáctico. En A. Medina. y F. Salvador (Coords.). Didáctica general. Madrid: Prentice Hall.

Casero, A. (2008). Propuesta de un cuestionario de evaluación de la calidad docente universitaria consensuado entre alumnos y profesores. Revista de Investigación Educativa, vol.26 (1), 25-44.

Del Rincón, D. et al. (1995). Técnicas de investigación en Ciencias Sociales. Madrid: Dykinson.

Fuchs, C. (1997). Sistema de evaluación y mejoramiento de desempeño. Revista El Diario. Escuela de Negocios de la Universidad Adolfo Ibáñez.

González, I. y López, A.B. (2010). Sentando las bases para la construcción de un modelo de 
evaluación de las competencias docentes del profesorado universitario. Revista de Investigación Educativa, vol.28 (2), 403-423.

Jornet, J; González, J. y Sánchez, P. (2014). Factores contextuales que influyen en el desempeño docente. Revista Iberoamericana de Evaluación Educativa, vol.7 (2), 185-195.

Jornet, J.; Villanueva P.; Suárez J. M. y Alfaro, I. (1988). Proyecto de implantación de un sistema de evaluación del profesorado en la Universidad de Valencia. En Universidad de Valencia. Consideraciones metodológicas sobre la evaluación y mejora de la docencia universitaria. Informes de Investigación Evaluativa, 1. Valencia: Universidad de Valencia.

Krueger, R.A. (1991). El grupo de discusión, guía práctica para la investigación aplicada. Madrid: Pirámide.

López, A.B.; González, I. y León, C. de (2014). Perfil de un buen docente. Aplicación de un protocolo de evaluación de las competencias del profesorado universitario. Revista Electrónica Interuniversitaria de Formación del Profesorado, vol.17 (1), 133-148.

López, A.B.; González, I. y León, C. de (2015). Un análisis factorial exploratorio para la construcción de un modelo de indicadores de evaluación docente universitaria. Cultura y Educación, 27 (2), 337-371.

Montoya, J.; Arbesú, I.; Contreras, G. y Conzuelo, S. (2014). Evaluación de la docencia universitaria en México, Chile y Colombia: Análisis de experiencias. Revista Iberoamericana de Evaluación Educativa, 7 (2), 15-42.

Prins, F.J.; Sluijsmans, M.A.; Kirschner, P.A. y Strijbos, J.W. (2005). Formative Peer Assessment in a CSCL Environment: a Case Study. Assessment \& Evaluation in Higher Education, 30 (4), 417-444.

Saravia, M.A. (2011). Calidad del Profesorado: Un modelo de competencias académicas. Barcelona: Octaedro.

Stake, R.; Contreras, G. y Arbesú, I. (2011). Evaluando la calidad de la universidad, particularmente su docencia. Perfiles Educativos, vol.23, 155-168.

Tejedor, F.J. (2003). Un modelo de evaluación del profesorado universitario. Revista de investigación Educativa, vol.21 (1), 157-182.

Tejedor, F.J. (2012). Evaluación del desempeño docente. Revista Iberoamericana de Evaluación Educativa, vol.5 (1), 318-327.

Tejedor, F.J. y García Valcárcel, A. (1996). La evaluación de la calidad de la docencia universitaria (en el marco de la evaluación institucional) desde la perspectiva del alumno. En F.J. Tejedor y J.L. Rodríguez Diéguez (Eds.), Evaluación Educativa. II. Evaluación institucional. (pp. 93122). Salamanca: Universidad de Salamanca.

Tejedor, F. J. y Jornet, J. M. (2008). La evaluación del profesorado universitario en España. Revista Electrónica de Investigación Educativa, Especial. Recuperado el 11 de agosto de 2015 desde http://redie.uabc.mx/NumEsp1/contenido- tejedorjornet.html

Universidad de Concepción (2011). Modelo Educativo. Concepción: Universidad de Concepción. Recuperado el 11 de agosto de 2015 desde http://docencia.udec.cl/wp-content/uploads/2014/05/ Modelo_educativo_opti.pdf

Universidad de Concepción (2015). Perfil docente UdeC. Recuperado el 11 de agosto de 2015 desde http://docencia.udec.cl/unidd/images/stories/documentos/PERFIL_DOCENTE.pdf

Universidad de Córdoba (2010). Programa Docentia-Córdoba. Recuperado el 11 de agosto de 2015 desde http://www.uco.es/organizacion/calidad/docentia/PDF/docordoba.pdf.

Zabalza, M.A. (2007). La enseñanza universitaria. El escenario y sus protagonistas. Madrid: Narcea. 
